\title{
Heat Transfer Modeling of Bipropellant Thrusters for using in Multidisciplinary Design Optimization Algorithm
}

\author{
A. Adami ${ }^{1}$, M. Mortazavi ${ }^{1}$, M. Nosratollahi ${ }^{2}$ \\ ${ }^{1}$ Amirkabir University of Technology, Tehran, Iran \\ Aha.aerospace@aut.ac.ir; mortazavi@aut.ac.ir \\ 2Malek e Ashtar University of Technology, Tehran, Iran \\ M_nosratollahi@sbu.ac.ir
}

\begin{abstract}
Modern designing methods such as multidisciplinary design optimization (MDO) need semi-accurate model of each discipline in preliminary design phase to analyze many configurations in a shorter elapsed time. One of the most important disciplines in multidisciplinary design optimization of bipropellant is thermal-control and cooling. Every cooling method such as regenerative, film and radiative cooling needs to predict heat transfer from combustion chamber. All known relations for determination of the convective heat transfer coefficient require many parameters especially wall temperatures, which is not accessible before cooling. Using such relations lead to implicit solutions and time consuming. In this paper, engineering model of heat transfer from combustion chamber is introduced for using in multidisciplinary design optimization. The presented algorithm is useful for every cooling method and needs few inputs. The algorithm analytically predicts convective and radiative heat flux from combustion chamber. The results have been compared with the experimental data as well as the numerical results for different geometries, $O / F$ ratios, propellants, and combustion chamber pressures. According to the results, presented algorithm can suitably predict the heat fluxes with error lower than15 percentage. The algorithm is not involved in implicit solution or numerical complexity and can be used in multidisciplinary design optimization methods that demands rapid heat transfer modeling.
\end{abstract}

Keywords: Heat Transfer, Heat Flux, Bipropellant, Multidisciplinary Design Optimization.

(C) Copyright 2015 Authors - This is an Open Access article published under the Creative Commons Attribution License terms (http://creativecommons.org/licenses/by/3.0). Unrestricted use, distribution, and reproduction in any medium are permitted, provided the original work is properly cited.

Date Received: 2014-11-02

Date Accepted: 2015-11-20

Date Published: 2015-12-21

\section{Nomenclature}

MDO Multidisciplinary design optimization

$\mathrm{O} / \mathrm{F} \quad$ Oxidant mass by fuel mass ratio

$\dot{q}_{h}^{\gamma} \quad$ convective heat flux

$\dot{q}_{r}^{\gamma} \quad$ radiative heat flux

$h_{\gamma} \quad$ convective heat coefficient

$T_{s} \quad$ wall temperature

$\varepsilon_{\gamma} \quad$ Radiative coefficient of hot gas

$\tilde{T} \quad$ mean temperature

$T_{1} \quad$ temperature before heat transfer

$T_{2} \quad$ temperature after heat transfer (real

temperature)

$C_{P} \quad$ specific heat at constant pressure

$R_{e} \quad$ Reynolds number

$R$ constant parameter of gas combustion products

$M_{g} \quad$ molecular weight

$K_{T} \quad$ temperature correction factor

$K_{X} \quad$ boundary layer correction factor

Pr Prandtl number

$M \quad$ Mach number

$\gamma \quad$ isentropic exponent

$A_{t} \quad R_{t} \quad$ Throat area, radius of throat

$\rho$ ' Density of gas

$Z_{c} \quad$ correction factor for area

$A_{\text {cross }}$ cross-area 


$\begin{array}{ll}A_{\text {comb }} & \text { combustion chamber cross section } \\ A_{\text {lat }} & \text { total lateral surface including nozzle surface } \\ \mu & \text { Viscosity coefficient } \\ T_{0} & \text { combustion temperature (T-core) } \\ \dot{m} & \text { Mass flow } \\ R_{\text {Throat Curve }} & \text { Curvature radius of the throat } \\ K_{\text {gas }} & \text { conductivity of combusted gases }\end{array}$

\section{Introduction}

Liquid thrusters are widely used in space applications especially for orbital maneuvers and launch vehicles. New missions demand new propulsions with lower cost. Additionally, new materials and propellants will improve the propulsion performances so, new bipropellant propulsions will be born. Modern designing methods such as multidisciplinary design optimization (MDO) are used to derive an optimum solution. Optimization of complex engineering systems has always been an integral part of the design. Due to the size and complexity of the systems, the design of a whole system is broken down into multiple disciplines. All disciplines should be simultaneously optimized to find a global optimum. Time consumption is a disadvantage and always tries to reduce the elapsed time. In preliminary design phase, semi-accurate model of each discipline is usually used to analyze many configurations in a shorter elapsed time. One of the most important disciplines in multidisciplinary design optimization of the bipropellant propulsion is thermal-control and cooling.

The purpose of a thermal-control is to maintain all thrusters' components within the allowable temperature. It is only obtained by control of heat fluxes from a combustion chamber. Because of high combustion temperatures (up to $3700 \mathrm{~K}$ ) and high heat flux rates (peak may go beyond $160 \mathrm{MW} / \mathrm{m}^{2}$ ) from the hot gases to the chamber wall, thruster cooling is a major design consideration. Two approaches are available to encounter [1], [2]. The first one is steady state method, which the heat transfer rate through combustion wall and temperature on the wall are constant, in other words, there is a thermal equilibrium. The second method is transient heat transfer method. For this method, there is no thermal equilibrium and the temperature on combustion wall continuously increases. The propulsion system has to be stopped before the temperature reaches the critical point. For continues thrust regime in space mission, steady state methods should be provided. Regenerative, transpiration, radiative, ablative and film cooling are generally used to control heat fluxes of combustion chamber and to provide suitable structure temperatures [3], [4].

Regenerative cooling is the most widely used method of cooling a high performance combustion chamber. It is accomplished by flowing high velocity coolant over the backside of the chamber hot gas wall to convectively cool the wall [5], [6]. The coolant, heated up by the hot liner, is then discharged into the injector and used as a propellant. Engines that have this kind of cooling are, for example, the AETUS II, RL10A, RD861Kand VINCI [7]. Ablative cooling uses a particular combustion gas-side wall material that is sacrificed, by melting, vaporization and chemical changes to dissipate heat. As a result, relatively cool gases flow over the wall surface, thus lowering the boundary-layer temperature and assisting the cooling process. Radiative cooling radiates the heat away from the surface of the outer chamber walls. An example of entirely radiation-cooled thruster is the RS-21 Mariner/Viking Orbiter spacecraft. Transpiration cooling is accomplished by introducing a coolant (gaseous or liquid) through porous chamber walls at a rate sufficient to maintain the desired combustion chamber wall temperature. This technique was applied to cool the injector faces of the J-2, RS-44 and SSME. Film cooling protects the chamber walls from excessive heat with a thin film of coolant. Coolant is injected through orifices near the injector or near the throat with special slots. This method has been widely used, in particular, for high heat fluxes [8]-[10].Sample engines were film cooling is applied are the SSME, Vulcain2, RD-171and RD-180.Combinations of mentioned methods are nowadays used [11] to increase the thermal performances. Combination of regenerative and film cooling is more interesting because of good performance without activity limitation.

Good thermal control needs good heat fluxes approximation. Nowadays, many numerical methods and commercial tools have been developed which try to improve modeling of heat fluxes. All of the methods and tools need many parameters and times to run. Such inputs may be available for existing thrusters but some required inputs are not accessible in preliminary design phases. Rapid heat transfer modeling is especially required for preliminary design phases. Although such an algorithm has lower accuracy but faster heat transfer modeling make a good possibility to analyze many configurations for deriving best performance. In this 
paper, engineering model for heat transfer will be introduced which can be applied in MDO algorithms.

\section{Heat Transfer Modelling}

Thermal-control of bipropellant thrusters is one of the most important disciplines in propulsion design algorithm. Hard coupling between thermal limitation and thruster's performance (such as pressure, mass flow, $0 / F$ ratio...) takes place because one of the propellant (usually fuel) is simultaneously involved in different disciplines [4], [12]. The largest part of the heat transfer from the hot chamber gases to the chamber walls is by convection and radiation. The amount of heat transfer by conduction is small and usually negligible in preliminary design phases.

Combustion products lose energy by convection $\dot{q}_{h}^{\gamma}$ and radiation $\dot{q}_{r}^{\gamma}$. Finally heat fluxes are emitted by radiation or be absorbed by some other coolant from lateral surface.

$$
\left\{\begin{array}{l}
\dot{q}_{h}^{\gamma}=h_{\gamma}\left(\tilde{T}-T_{s}\right) \\
\dot{q}_{r}^{\gamma}=\varepsilon_{\gamma} \sigma T_{01}^{4} \\
\sigma=5.6697 \times 10^{-8} \frac{W}{\left(m^{2} \cdot K^{4}\right)} \\
\tilde{T}=\frac{T_{1}+T_{2}}{2}
\end{array}\right.
$$

$T_{s}$ is wall temperature, $\tilde{T}$ is mean temperature and $T_{2}$ is a temperature after heat transfer (real temperature). $T_{2}$ is calculated by solving the energy equilibrium . Prediction of convective heat coefficient ( $h_{\gamma}$ ) is most important part of thermal modeling of every combustion chamber. A few analytical models are available to predict $h_{\gamma}$. Bartz at 1963 [13] proposed empirical correlations (2) for prediction of $h_{\gamma}$ which is widely used. There was no offer for $\dot{q}_{r}^{\gamma}$. $h_{\gamma}=0.026 \mu^{0.2} C_{P}^{0.4}\left(\frac{K_{\text {gas }}}{\mu}\right)^{0.6}\left(\frac{\dot{m}_{\text {Throat }}^{0.8}}{A^{0.9}}\right)\left(\frac{\pi D_{t}}{4 R_{\text {Throat Curve }}}\right)^{0.1}$

$\dot{q}_{r}^{\gamma} \sim 0$

Pavli and et al. at 1966 [14] presented relation (3) which was included the Reynolds number $R_{e}$ and two correction factors to improve the behavior of the original Bartz equation. A temperature correction factor $K_{T}$ had been added, taking into account that the new reference temperature is calculated halfway between the wall and the free stream static temperature. Moreover, since the geometric reference parameter in the original Bartz equation was the throat diameter, a further correction factor $K_{X}$ had been added for the consideration of the boundary layer growth in the cylindrical part and at nozzle.

$$
\begin{aligned}
& h_{\gamma}=0.023 \mathrm{Re}^{-0.2} \operatorname{Pr}^{-0.6} C_{P}\left(\frac{\dot{m}}{A_{\text {Cross }}}\right) K_{T} K_{X} \\
& K_{T}=\left(\frac{T_{\gamma}}{\tilde{T}}\right)^{e} \\
& K_{X}=\left(\frac{R}{R_{\text {Throat Cuve }}}\right)^{f} \\
& \tilde{T}=\frac{T_{0}+T_{\text {wall }}}{2} \\
& \dot{q}_{r}^{\gamma} \sim 0
\end{aligned}
$$

Huzel and et al. at 1992 [1] proposed equations (4) for prediction of $h_{\gamma}$ and $\dot{q}_{r}^{\gamma}$. More quality of flow including Mach number and pressure were taken into account.

At 2001, ref [15] presented relation (5) based on two mentioned correction factors and the effect of cross section was augmented. 


$$
\begin{aligned}
& h_{\gamma}=\left[\frac{0.026}{D_{t}^{0.2}}\left(\frac{\mu^{0.2} C_{P}}{\operatorname{Pr}^{0.6}}\right)\left(\frac{P_{0} g}{c^{*}}\right)^{0.8}\left(\frac{D_{t}}{R_{\text {Throat Curve }}}\right)^{0.1}\left(\frac{A_{t}}{A}\right)^{0.9} \sigma\right. \\
& \sigma=\frac{1}{\left[0.5 \frac{T_{w g}}{T_{0}}\left(1+\frac{\gamma-1}{2} M^{2}\right)+0.5\right]^{0.68}\left[1+\frac{\gamma-1}{2} M^{2}\right]^{0.12}} \\
& \dot{q}_{r}^{\gamma} \sim 0 \\
& h_{\gamma}=0.026 \mu^{0.2} C_{P}^{0.4}\left(\frac{K_{\text {gas }}}{\mu}\right)^{0.6}\left(\frac{\dot{m}_{\text {Throat }}^{0.8}}{A^{0.9}}\right)\left(\frac{\pi D_{t}}{4 R_{\text {Throat Cuvve }}}\right)^{0.1} K_{T} K_{X} \\
& K_{T}=\left(\frac{T_{\gamma}}{\tilde{T}}\right)^{a}, K_{X}=\left(\frac{R}{R_{\text {Throat Curve }}}\right)^{b}, \tilde{T}=\frac{T_{0}+T_{\text {wall }}}{2} \\
& \dot{q}_{r}^{\gamma} \sim 0
\end{aligned}
$$

Finally Sutton at reference [16] proposed equations (3) for prediction of $h_{\gamma}$ and $\dot{q}_{r}^{\gamma}$.

$$
\begin{aligned}
& h_{\gamma}=\frac{k_{d}}{d} 0.0162 \operatorname{Pr}_{g}^{0.82} \operatorname{Re}_{g}^{0.82}\left(\frac{T_{a w}}{T_{w g}}\right)^{0.35} \\
& \dot{q}_{r}^{\gamma}=\dot{q}_{r}^{C O_{2}}+\dot{q}_{r}^{H_{2} O} \\
& \dot{q}_{r}^{\mathrm{CO}_{2}}=3.5 \sqrt[3]{P_{C O_{2}} L_{e}}\left[\left(\frac{T_{a w}}{100}\right)^{0.35}-\left(\frac{T_{w g}}{100}\right)^{0.35}\right] \\
& \dot{q}_{r}^{H_{2} \mathrm{O}}=3.5 P_{C O_{2}}^{0.8} L_{e}^{0.6}\left[\left(\frac{T_{a w}}{100}\right)^{3}-\left(\frac{T_{w g}}{100}\right)^{3}\right]
\end{aligned}
$$

All mentioned equations need wall temperature to calculate heat fluxes. In fact, correction factor usually have been developed based on temperature ratio. For rapid thermal modeling of combustion chamber, wall temperature should be a solution of energy equations and if $h_{\gamma}$ be involved in wall temperature then implicit solution is derived instead of direct solution. Furthermore, above equations, need many inputs $\left(a, b, e, f, T_{\gamma}, T_{\text {wall }}\right)$ that are not usually available in preliminary design phase.

\section{Rapid Heat Transfer Modeling}

According to the mentioned relations, $h_{\gamma}$ is a function of following terms.

$$
h_{\gamma}=f\left(\operatorname{Re}, \operatorname{Pr}, C_{P}, \dot{m}, A, \mu, T\right)
$$

Compression between equation (7) and convective heat coefficient of flat plat offers equation (8):

$$
h_{\gamma}=Z_{c} 0.5 \rho^{a} V^{b} C_{P}^{c} \mu^{d} \operatorname{Pr}^{e}
$$

Using mass flow equilibrium through the combustion and nozzle leads to equation (9).

$$
\left.\begin{array}{l}
a=1 \\
b=1
\end{array}\right\} \Rightarrow 0.5 \rho^{a} V^{b}=\frac{\dot{m}}{2 A_{\text {cross }}}
$$

$A_{\text {cross }}$ is cross-area of every section and $\dot{m}$ is related mass flow. Other exponents and correction factors are suggested as below.

$\left\{\begin{array}{l}c=1 \\ d=0.3 \\ e=-\frac{2}{3} \\ Z_{c}=\frac{A_{\text {comb }}}{A_{\text {lat }}}\end{array}\right.$

Where, ${ }^{A}{ }_{\text {comb }}$ is combustion chamber cross section and $A_{\text {lat }}$ is total lateral surface including nozzle surface. New correction factor presents the effect of geometry (length and diameter) and 2-D heat transfer in structure. Replacing equations (9) and (10) in equation (8) finally leads to equation (11).

$$
\begin{aligned}
& h_{\gamma}=Z_{c} 0.5 \rho_{\text {comb }} V_{\text {flow }} C_{P} \mu^{0.3} \operatorname{Pr}^{-\frac{2}{3}} \\
& \Rightarrow h_{\gamma}=Z_{c} \frac{\dot{m}_{\text {propellout }}}{2 A} C_{P} \mu^{0.3} \operatorname{Pr}^{-\frac{2}{3}} \\
& Z_{c}=\frac{\pi R_{\text {comb }}^{2}}{A_{\text {lat }}}
\end{aligned}
$$

$\operatorname{Pr}$ is the Prandtl number, ${ }^{\mu}$ is viscosity, $C_{P}$ is specific heat at constant volume and $A$ is cross section of flow $\left(A^{*}<A<A_{\text {comb }}\right.$ )and $Z_{c}$ is correction factor depended on combustion chamber geometry. Equation 
(12) presents supplementary relations [16], [4] when exact parameters of flow are not available.

$R$ is a constant parameter of gas combustion products, $\gamma_{\text {is an isentropic exponent, }} M_{g}$ is molecular weight and $T_{0}$ is combustion temperature (T-core). $R$, $\gamma_{\text {and }} T_{0}$ can be derived from [17] for every propellant. Finally, heat transfer modeling can summarily be presented as figure (1).

$$
\begin{aligned}
& \varepsilon_{\gamma} \sim 0.1 \\
& C_{P}=\frac{\gamma R}{\gamma-1} \\
& \operatorname{Pr}=\frac{4 \gamma}{9 \gamma-1} \\
& \mu=1.78 \times 10^{-7} M_{g}^{0.5} T_{0}^{0.6}
\end{aligned}
$$

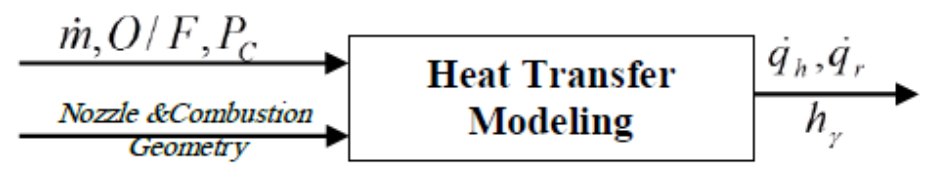

Figure 1. Heat Transfer Discipline and Input-Output Flow Data.

\section{Validation}

Validation of heat transfer usually concentrated in prediction of $\dot{q}_{h}$ and $\dot{q}_{r}$. Validation has been done by numerical results and experimental data [18], [15]. The results of the presented algorithm and the results of numerical methods have been compared at table 1 and table 2 for following two cases.

Case-1

$$
\begin{aligned}
& A_{\text {comb }}=0.0172 \mathrm{~m}^{2} \\
& A_{\text {lat }}=0.3403 \mathrm{~m}^{2} \\
& O / F=2.5\left(\mathrm{~N}_{2} \mathrm{O}_{4}-U D M H\right)
\end{aligned}
$$

Thrust $=4000(N)$

$P_{c}=30($ bar $)$

$T_{s}=835(K)$

$\dot{m}=1.31 \frac{\mathrm{kg}}{\mathrm{s}}$

\section{Case-2}

$$
\begin{aligned}
& A_{\text {comb }}=0.0226 \mathrm{~m}^{2} \\
& A_{\text {lat }}=0.1675 \mathrm{~m}^{2} \\
& O / F=1.5\left(\mathrm{~N}_{2} \mathrm{O}_{4}-U D M H\right)
\end{aligned}
$$

$$
\text { Thrust }=4000(N)
$$$$
P_{c}=25(\text { bar })
$$$$
T_{s}=835(K)
$$$$
\dot{m}=1.41 \frac{\mathrm{kg}}{\mathrm{s}}
$$

According to the results, presented model can suitably (Error < 15percentage) predict heat transfer. Experimental results have been presented as dimensionless for following conditions:

$O / F=6.0\left(\mathrm{O}_{2}-\mathrm{H}_{2}\right)$

$P_{c}=60($ bar $)$

$T_{\text {s- comb }}=550(K)$

$T_{\text {s- Throat }}=660(K)$

Figure (2) shows the comparison between the results of presented algorithm (14 sections) and experimental data.

Table 1. Results comparison for the case-1.

\begin{tabular}{|l|c|c|c|c|c|}
\hline & $\begin{array}{c}\mu \\
\text { atcombustion }\end{array}$ & $\begin{array}{c}\dot{q}_{h} \\
(\text { combustion })\end{array}$ & $\begin{array}{c}\dot{q}_{r} \\
(\text { combustion })\end{array}$ & $\begin{array}{c}\dot{q}_{h} \\
(\text { Throat })\end{array}$ & $\begin{array}{c}\dot{q}_{r} \\
(\text { Throat })\end{array}$ \\
\hline $\begin{array}{l}\text { Bartz } \\
\text { relation }\end{array}$ & $1.03 \times 10^{-4} \frac{\mathrm{kg}}{\mathrm{m} \cdot \mathrm{s}}$ & $1.95\left(\frac{M W}{\mathrm{~m}^{2}}\right)$ & $0.43\left(\frac{\mathrm{MW}}{\mathrm{m}^{2}}\right)$ & $28.70\left(\frac{M W}{\mathrm{~m}^{2}}\right)$ & $0.29\left(\frac{M W}{\mathrm{~m}^{2}}\right)$ \\
\hline $\begin{array}{l}\text { Presented } \\
\text { Algorithm }\end{array}$ & $1.15 \times 10^{-4} \frac{\mathrm{kg}}{\mathrm{m} \cdot \mathrm{s}}$ & $1.68\left(\frac{M W}{\mathrm{~m}^{2}}\right)$ & $0.45\left(\frac{M W}{\mathrm{~m}^{2}}\right)$ & $33.02\left(\frac{M W}{\mathrm{~m}^{2}}\right)$ & $0.32\left(\frac{M W}{\mathrm{~m}^{2}}\right)$ \\
\hline Error & $10 \%$ & $14 \%$ & $5 \%$ & $15 \%$ & $10 \%$ \\
\hline
\end{tabular}

Table 2. Results comparison for the case-2.

\begin{tabular}{|l|c|c|c|c|c|}
\hline $\begin{array}{c}\mu \\
\text { atcombustion }\end{array}$ & $\begin{array}{c}\dot{q}_{h} \\
(\text { combustion })\end{array}$ & $\begin{array}{c}\dot{q}_{r} \\
\text { (combustion) }\end{array}$ & $\begin{array}{c}\dot{q}_{h} \\
\text { (Throat) }\end{array}$ & $\begin{array}{c}\dot{q}_{r} \\
(\text { Throat })\end{array}$ \\
\hline $\begin{array}{l}\text { Bartz } \\
\text { relation }\end{array}$ & $0.84 \times 10^{-4} \frac{\mathrm{kg}}{\mathrm{m} . \mathrm{s}}$ & $1.42\left(\frac{M W}{\mathrm{~m}^{2}}\right)$ & $0.24\left(\frac{M W}{\mathrm{~m}^{2}}\right)$ & $23.70\left(\frac{M W}{\mathrm{~m}^{2}}\right)$ & $0.14\left(\frac{M W}{\mathrm{~m}^{2}}\right)$ \\
\hline $\begin{array}{l}\text { Presented } \\
\text { Algorithm }\end{array}$ & $0.86 \times 10^{-4} \frac{\mathrm{kg}}{\mathrm{m} . \mathrm{s}}$ & $1.22\left(\frac{M W}{\mathrm{~m}^{2}}\right)$ & $0.22\left(\frac{M W}{\mathrm{~m}^{2}}\right)$ & $20.97\left(\frac{M W}{\mathrm{~m}^{2}}\right)$ & $0.16\left(\frac{M W}{\mathrm{~m}^{2}}\right)$ \\
\hline Error & $3 \%$ & $14 \%$ & $9 \%$ & $11 \%$ & $15 \%$ \\
\hline
\end{tabular}

According to the results, algorithm can suitably predict heat fluxes after complete combustion (better than Bartz). It should be noted that the presented algorithm do not need wall temperature to calculate $h_{\gamma}$ and wall temperature is only needed to calculate the heat fluxes. Calculated heat fluxes by presented algorithm can 
be used for every cooling method. It is clear that real combustion chamber temperature should be sequentially updated (instead of adiabatic temperature) if energy losing occurs (radiation, ablation ...).

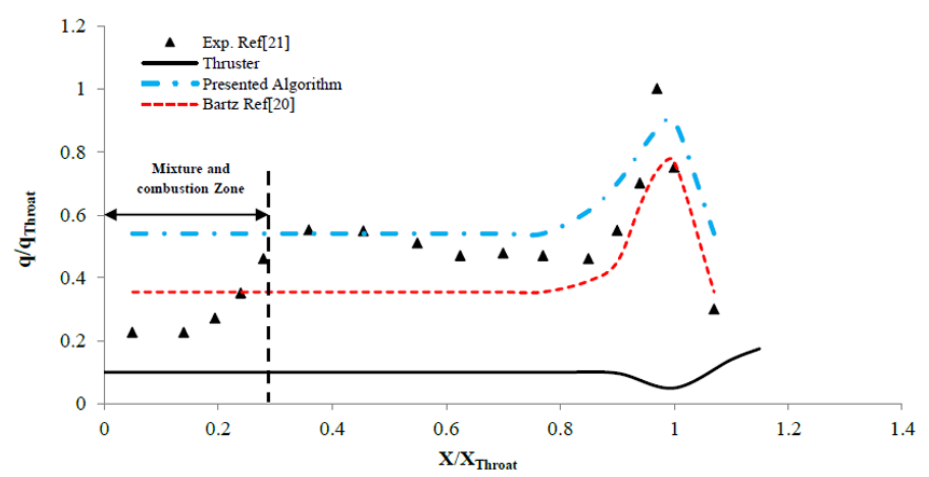

Figure 2. Results comparison between presented algorithm and experimental data of ref [15], [18].

\section{Summary and Results}

In this paper, heat fluxes modeling of combustion chamber has been introduced. Previous relations such as Bartz and Pavli have been reviewed that need some parameters, which are not available for new thrusters in preliminary design phase. New relations for convective and radiative heat fluxes have been presented which can be applied in MDO. Presented algorithm derives the convective heat coefficient as a function of mass flow, $\mathrm{O} / \mathrm{F}$ ratio, pressure, and cross sections. General advantages of the presented algorithm are summarized as applicability for any propellant and thrust value, analytical solution and demanding a few inputs. Results have been compared with numerical methods as well as experimental data for different propellant, $\mathrm{O} / \mathrm{F}$ ratio, combustion pressure, and wall temperature. According to the results, algorithm can suitably predict heat fluxes after complete combustion. Therefore, algorithm can be applied in preliminary design phase.

\section{References}

[1] D. K. Huzel and D. H. Huang, Modern Engineering for Design of Liquid-Propellant Rocket Engines, Washington: American Institute of Aeronautics and Astronautics, 1992.

[2] M. Bai, "Numerical Evaluation of Heat Transfer and Pressure Drop in Open Cell Foams," MSc thesis, Florida: University of Florida, 2007.

[3] M. Trotti, "Modelling of liquid film cooling in a GOX/Kerosene rocket combustion chamber," Ph.D. dissertation, Italy: Politecnico Di Milano, 2011-2012.
[4] A. Amirhossein, "Multidicsiplinary Design Optimization of Reentry Vehicle considering Guidance Algorithm," Ph.D. dissertation, Iran: Amirkabir University of Technology, 2015.

[5] Y. Torres, L. Stefanini and D. Suslov, "Influence of Curvature in RegenerativeCooling System of Rocket Engine," Progress in Propulsion Physics, vol. 1, pp. 171184, 2009.

[6] M. Pizzarelli, F. Nasuti and M. Onofri, "Trade-off analysis of high-aspect-ratio-cooling-channels for rocket engines," International Journal of Heat and Fluid Flow, vol. 44, pp. 458-467, 2013.

[7] M. E. Boysan, "Analysis of regenerative cooling in liquid preopellant rocket engines," MSc thesis, Turkey: Middle East Technical University, 2008.

[8] R. Arnold, D. I. Suslov and O. J. Haidn, "Film cooling of accelerated flow in a subscale combustion chamber," Journal of propulsion and power, vol. 25, no. 2, pp. 443451, 2009.

[9] R. Arnold, D. I. Suslov and O. J. Haidn, "Film cooling in a high-pressure subscale combustion chamber," Journal of propulsion and power, vol. 26, no. 3, pp. 428-438, 2010. [10] Babaee and Hessam, "Analysis and Optimization of Film Cooling Effectiveness," Ph.D. dissertation, Louisiana: Louisiana State University, 2013.

[11] O. Knab, M. Frey, J. Görgen, C. Maeding, K. Quering and D. Wiedmann, "Progress in Combustion and Heat Transfer Modelling in Rocket Thrust Chamber Applied Engineering," in 45th AIAA/ASME/SAE/ASEE Joint Propulsion Conference \& Exhibit, Colorado, 2009.

[12] H. Darabi, J. Roshanian and H. Zare, "Design of liquid-propellant engine using collaborative optimization and evolutionary algorithms," in Proceedings of the Institution of Mechanical Engineers, Part G: Journal of Aerospace Engineering, Spain, 2014.

[13] D. R. Bartz, "Turbulent boundary-layer heat transfer from rapidly accelerating Wow of rocket combustion gases and of heated air," Jet Propulsion Laboratory, California, Tech. Rep. NASA-CR-62615, 1963.

[14] A. J. Pavli, J. K. Curley, P. A. Masters and R. M. Schwartz, "Design and Cooling Performance of a Dump Cooled Rocket Engine," NASA, Washington, Tech. Memo. D-3532, 1966.

[15] T. D. Astrium, "Final report on data correlation and evaluation of test results phase 1 part 1," Astrium, Contract GSTP-2-TN-06-Astrium, Final Rep., 2001.

[16] G. P. Sutton and O. Biblarz, Rocket Propulsion Elements, New Jersey: John Willy \& Sons Inc., 2010.

[17] S. Gordon and B. J. McBride, Computer program for calculation of complex chemical equilibrium 
compositions and applications, Ohio: NASA, Tech. Rep., 1994.

[18] F. Di Matteo, "Modelling and Simulation of Liquid Rocket Engine Ignition Transients," Ph.D. dissertation, Rome: Sapienza University of Rome, 2011. 